\title{
Looking ahead to Neptune
}

Pasadena

VOYAGER 2, now old and far away, has nonetheless given the National Aeronautics and Space Administration (NASA) one bright spot in an otherwise gloomy year. So, on 19 September, NASA said thank you by handing out a spate of awards to Voyager team members, including the agency's highest award, the Distinguished Service Medal, to Richard Laeser, Voyager's project manager since 1981.

Under Laeser's direction, Voyager 2 was essentially re-engineered from the ground while on its way from Saturn to Uranus. The result was a much more fruitful encounter than anyone could have predicted when the spacecraft left Earth in 1977. Now Voyager team members are looking towards the encounter with Neptune in 1989 , and in the next few months Laeser and his colleagues at the Jet Propulsion Laboratory (JPL) face some tough decisions about the character of this next planetary visit.

The main issue that Voyager's planners are wrestling with concerns the spacecraft's trajectory. A close approach is best for imaging, but makes problems for radio science. If Voyager passes too close to Neptune, it could experience atmospheric drag, something it is not built to withstand. Even a small amount of drag could affect the spacecraft's attitude.

Another difficulty in too close an approach to Neptune is the possibility of an unintentional encounter with one of Neptune's recently discovered fragmentary rings. That, says project scientist Edward Stone, is unlikely, since the ring arcs account for only about 10 per cent of an entire ring, and are quite narrow. Even hitting them intentionally would not be easy.

The decision about how close to come to Neptune will have a profound effect on

\section{Deltaplan defence line completed}

Waalre, The Netherlands

ON the night of 1 February 1953 more than 1,800 people lost their lives when the North Sea breached the Dutch sea defences, flooding large areas of south-western Holland. To stop such a disaster recurring, a remarkable civil engineering project - the Deltaplan - was drawn up, and the last link in the defensive chain is about to be officially opened.

The overall effect of the Deltaplan is to reduce the exposed coastline of Holland by some $700 \mathrm{~km}$. The last link, a storm surge barrier across the Eastern Scheldt estuary, is the biggest single project in the plan. Not only is it the most costly project, but also the most controversial. The original plan had involved a closed dam across the 9-km- wide estuary, but pressure from conservation groups and the fisheries industry led to the change to a 3-km-wide storm-surge barrier that allows the tide to flow normally but permits the closure of the estuary if floods are predicted.

In its final form the barrier is made up of 66 piers placed in three tidal channels; four times a day 800 million cubic metres of water passes through the $\mathbf{6 3}$ gates in the barrier. Model studies suggest that there will be considerable changes in the ecology of the area if the barrier has to be closed. Even without closures, there are worries about the sensitive oyster beds in the east of the estuary, where the mean tidal range will be reduced from 3.5 to 2.7 metres. Casper Schuuring

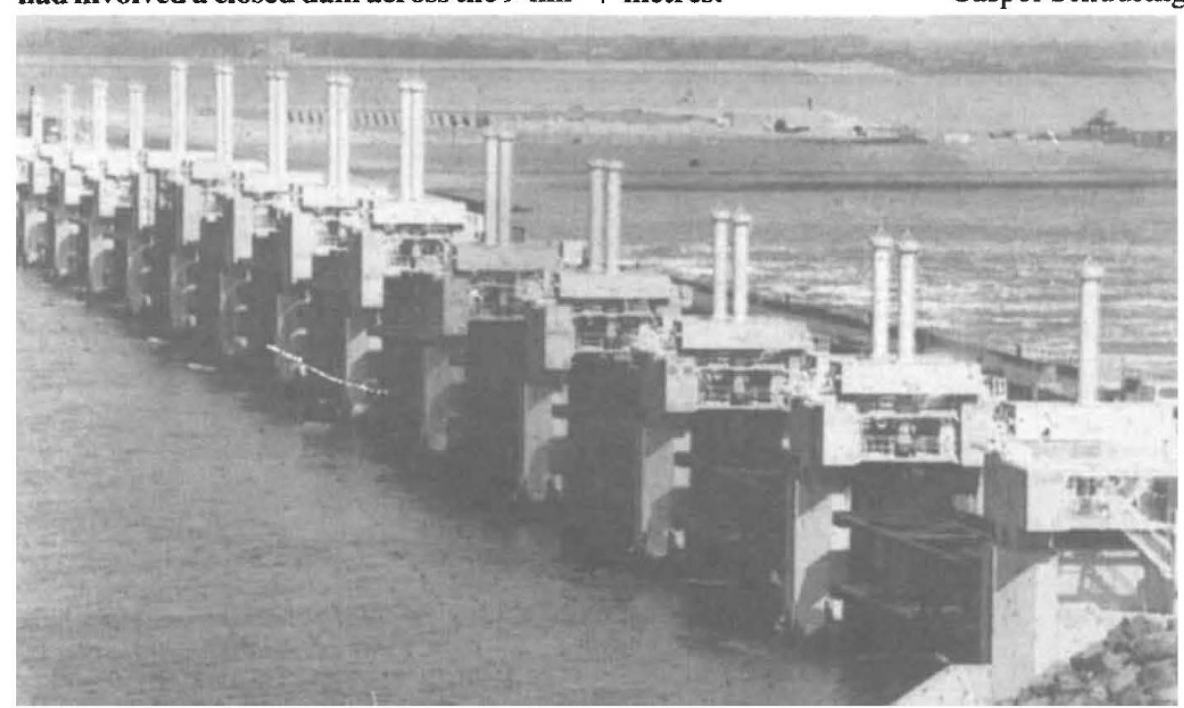

Some of the 63 gates in the Eastern Scheldt barrier. A public road runs along the top of the barrier, 12 metres above sea level.

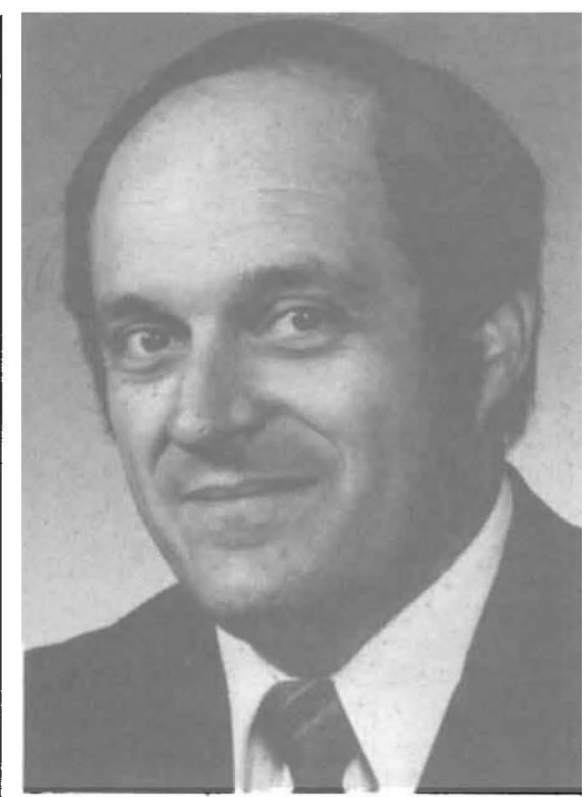

Laeser: re-engineered Voyager between planets.

how near the spacecraft will approach Neptune's large moon Triton. Measuring from the centre of the planet, a flyby of Neptune at $27,500 \mathrm{~km}$ would bring Voyager as close as $25,000 \mathrm{~km}$ from the centre of Triton. But a Neptune flyby at 32,300 $\mathrm{km}$ puts Voyager $68,000 \mathrm{~km}$ from Triton. The advantage of a more distant approach is that there would be more margin for error as the spacecraft tracks the moon's limb with its radio antennas.

The radio occultation experiment will provide details of the planet's atmosphere. At Triton, a far approach also gives a benefit to the radio science team, but complicates the timing of a second experiment using scattered ultraviolet light from a solar occultation. A close approach to Triton would not only give better picture opportunities, but also allow an infrared investigation of Triton's atmosphere. A similar experiment earlier in the mission discovered hydrocarbons in the atmosphere of Saturn's moon Titan.

Laeser says that a decision on the trajectory will be made in the next few months. The sequence of events at the encounter will be planned on a second-to-second basis as the spacecraft makes its closest approach to the planet and moon.

The Voyager team now consists of 160 people at the Jet Propulson Laboratory, down from a peak of 200 earlier in the year during the Uranus encounter. Laeser expects that number to stay fairly stable until just before the Neptune encounter.

Despite NASA's budgetary uncertainties, Laeser expects Voyager to get the funds it needs to achieve all its objectives at Neptune. Laeser's confidence comes from the programme's good track record of sticking to its budget. Besides, he believes NASA is unlikely to do anything that will dim the brightest star in its currently murky firmament. Joseph Palca 